\title{
Intensity-modulated radiotherapy combined with iodine-125 seed implantation in non-central recurrence of cervical cancer: A case report and literature review
}

\author{
HU FENG $^{1}$, YUANYUAN HU ${ }^{1}$, PENG JIN $^{1}$, XIANGKUAN MENG $^{1}$, YUBING CHEN ${ }^{1}$ and HONGMEI ZHANG ${ }^{2}$ \\ ${ }^{1}$ Department of Radiotherapy, Second Hospital of Jilin University, Changchun, Jilin 130041; \\ ${ }^{2}$ Scientific Research Center, China-Japan Union Hospital of Jilin University, Changchun, Jilin 130033, P.R. China
}

Received June 5, 2016; Accepted March 3, 2017

DOI: $10.3892 / \mathrm{ol} .2017 .6680$

\begin{abstract}
Recurrent cervical cancer is a clinically complex disease that is difficult to treat. There are numerous treatment options, but the results achieved by each are poor. External-beam radiation therapy of the pelvic lymph drainage area, in combination with intracavitary afterloading or the interstitial implantation of a radiation source (i.e., brachytherapy), are the current standard radiotherapy regimens used in high-risk clinical targets. However, there are few reports concerning the use of iodine-125 $\left({ }^{125} \mathrm{I}\right)$ seed implantation brachytherapy in recurrent cervical cancer, and the effects of treatment and adverse reactions have not yet been systematically evaluated. In the present study one such case is reported, in which the patient was successfully treated with intensity-modulated radiotherapy (IMRT) in combination with ${ }^{125}$ I seed implantation. The patient, a 47-year-old woman, was initially diagnosed with International Federation of Gynecology and Obstetrics stage IB1 cervical cancer, and received a radical hysterectomy, left lateral adnexectomy and pelvic lymph node dissection. A follow-up examination 23 months later revealed vaginal invasion and a solitary lump in the cervical stump with a maximum diameter of $38 \mathrm{~mm}$. The patient was subsequently diagnosed with recurrent cervical cancer and was treated with six cycles of docetaxel
\end{abstract}

Correspondence to: Professor Yubing Chen, Department of Radiotherapy, Second Hospital of Jilin University, 218 Ziqiang Street, Changchun, Jilin 130041, P.R. China

E-mail: yb0707@163.com

Professor Hongmei Zhang, Scientific Research Center, China-Japan Union Hospital of Jilin University, 126 Xiantai Street, Changchun, Jilin 130033, P.R. China

E-mail: gzzhmei@163.com

Abbreviations: IMRT, intensity-modulated radiotherapy; PFS, progression-free survival

Key words: cervical cancer, non-central recurrence, interstitial iodine-125 seed implantation, intensity-modulated radiotherapy and nedaplatin chemotherapy, alongside IMRT and interstitial ${ }^{125}$ I seed implantation. At the point of manuscript submission, the patient's progression-free survival time was 33 months and long-term adverse reactions were acceptable. The response of this patient indicates that ${ }^{125} \mathrm{I}$ seed implantation could be used as a complementary treatment for recurrent cervical cancer and may also prove to be a reliable means for the comprehensive treatment of primary cervical cancer, as the patient had characteristics similar to primary cervical cancer, although this hypothesis could not be confirmed in the present study.

\section{Introduction}

Cervical cancer is the fourth most common malignant tumor in women and is responsible for the fourth-highest mortality rate (1). Early-stage cervical cancer can be cured with comprehensive treatment, which can include surgery, radiotherapy and chemotherapy, among others. However, approximately one-third of patients suffer recurrence, $75 \%$ of whom experience it within 2 years of treatment (2). Treatment of recurrent cervical cancer is effective in only $25 \%$ of cases and the median survival time is only 12 months (3). If the recurrence is local and the patient has no history of prior radiotherapy and cannot undergo surgical resection, radiotherapy is be used in combination with platinum-based chemotherapy. Brachytherapy can also be used, for cases where the tumor did not entirely subside (4).

Cervical cancer brachytherapy primarily involves intracavitary afterloading and the interstitial implantation of a radiation source. Broadly speaking, interstitial radioactive seed implantation is also included within the scope of brachytherapy, although it is not recommended as a routine treatment of cervical cancer under certain guidelines. The present case concerning non-central recurrence of cervical cancer was treated with intensity-modulated radiotherapy (IMRT) in combination with chemotherapy and interstitial iodine-125 $\left({ }^{125} \mathrm{I}\right)$ seed implantation. At the point of submission of the present manuscript, the progression-free survival (PFS) time had reached 26 months. This suggests that interstitial ${ }^{125}$ I seed implantation can be used as a complementary treatment for recurrent cervical cancer and, as the patient had characteristics 
similar to primary cervical cancer, may even have potential as a treatment for primary cervical cancer.

\section{Case report}

A 47-year-old woman presented to the Department of Gynecology of Changchun Central Hospital (Changchun, China) in July 2011 with contact vaginal bleeding. A diagnosis of cervical squamous cell carcinoma was reached by cervical biopsy and pathological analysis. The analysis revealed irregular cell morphology, large and irregular cell nuclei and cancer cell nests in stroma. The patient underwent a radical hysterectomy, left lateral adnexectomy and pelvic lymph node dissection on August 4, 2011. A postoperative pathological microscopic examination of a hematoxylin and eosin (H\&E)-stained tissue sample revealed an irregular cell shape, with large, irregular and deeply stained nuclei, and single keratinocytes, further confirming the diagnosis of squamous cell carcinoma (Fig. 1A and B). No cancerous cells were found in the vaginal stump or parametrial tissue and no evidence of metastasis was found in selected lymph node samples (5 lymph nodes were sampled per group). No further treatments, including radiotherapy and chemotherapy, were administered following surgery.

Written informed consent was obtained from the patient for publication of this case report and any accompanying images, in addition to permission from the Ethics Committee of the Second Hospital of Jilin University (Changchun, China).

The patient presented to the Department of Radiotherapy of the Second Hospital of Jilin University in July 2013 (23 months after surgery) with lower abdominal pain and abnormal vaginal discharge containing small amounts of blood. Liquid-based cervical cytology indicated a high-grade squamous intraepithelial lesion. An H\&E-stained cervical stump biopsy revealed cells with atypia and dark nuclei, supporting the diagnosis of squamous cell carcinoma (Fig. 1C and D). Pelvic magnetic resonance imaging (MRI) revealed an oval iso-T1 and long $\mathrm{T} 2$ signal shadow at the vaginal level, with a maximum lesion diameter of $\sim 38 \mathrm{~mm}$, departing from the center of the vagina by $\sim 40 \mathrm{~mm}$. An enhanced scan showed an evident heterogeneous enhancement (Fig. 2A). Levels of the serum tumor marker cancer antigen 125 (CA125) were $286.3 \mathrm{U} / \mathrm{ml},>8$ times higher than the normal limit $(\leq 35 \mathrm{U} / \mathrm{ml})$. The most common sites of metastases, including the lungs, liver and bone, exhibited no abnormalities. Thus, the case was diagnosed as recurrent cervical cancer.

Between July and August 2013 (23-24 months after surgery), the patient was treated with 2 cycles of intravenous docetaxel $(120 \mathrm{mg})$ and nedaplatin $(100 \mathrm{mg})$ at a 21 -day interval. Following this, pelvic MRI was performed, which showed a round iso-T1 iso-T2 signal shadow at the cervical stump level. The diameter of the shadow was $\sim 22 \mathrm{~mm}$, departing from the center of the vagina by $\sim 30 \mathrm{~mm}$. Enhanced MRI showed a heterogeneous enhancement with an uneven distribution of microvessels (Fig. 2B). The size of the shadow was markedly decreased when compared with that prior to treatment.

Between September and November 2013 (25-27 months after surgery), the patient was treated with external-beam radiation therapy, which ran concurrent with the previously described chemotherapy cycles. Following this, the patient
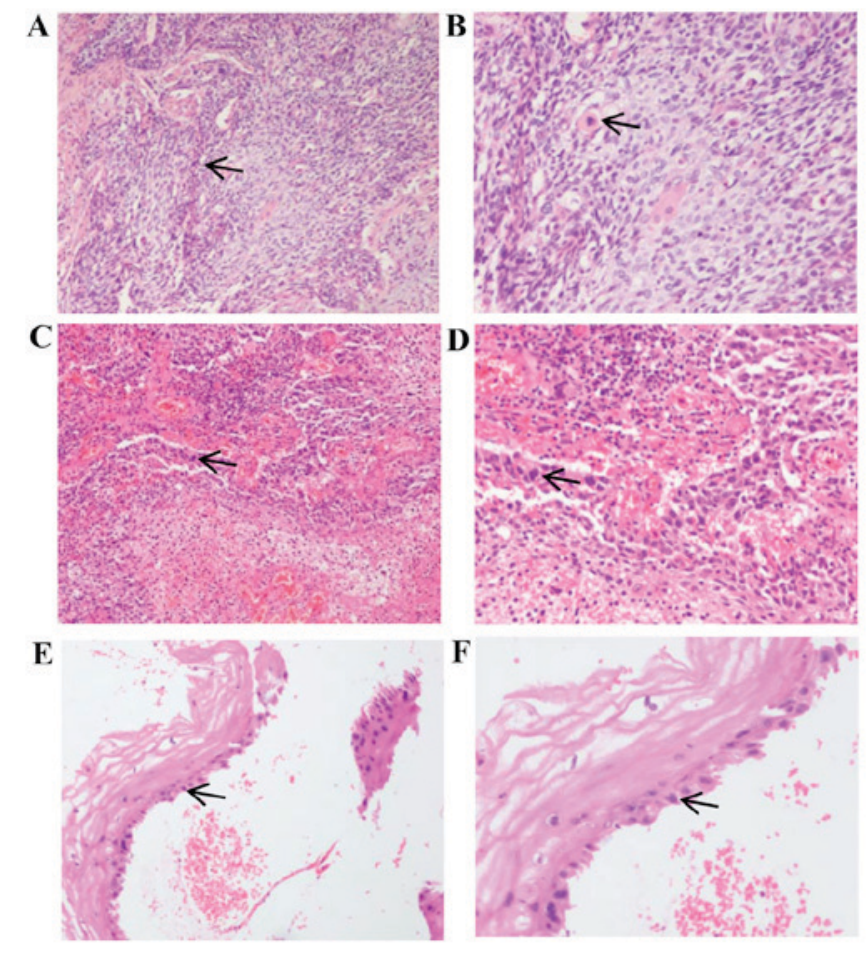

Figure 1. H\&E staining of cervical and recurrent tumor tissues. (A) Magnification x100 and (B) x200 images of H\&E staining of initially diagnosed cervical tissues. Microscopy reveals an irregular cell shape, large, irregular and deeply stained nuclei, and single keratinocytes, confirming the diagnosis of squamous cell carcinoma. (C) Magnification x100 and (D) X200 images of H\&E staining of cervical stump tissue from recurrent cancer. At the higher magnification in (D), tumor cells can be observed to invade the stroma of the cervix. The cells have atypia and dark nuclei, supporting the diagnosis of squamous cell carcinoma. (E) Magnifcation x100 and (F) x200 images of $\mathrm{H} \& \mathrm{E}$ staining of the cervical stump following external-beam irradiation, biopsy and chemotherapy. The squamous cells exhibit large, blurred nuclei, which are considered to be reactive changes caused by radiotherapy. Arrows indicate squamous cells in each image. H\&E, hematoxylin and eosin.

was treated with another 2 cycles of chemotherapy. Again, the chemotherapy program was 2 cycles of intravenous docetaxel $(120 \mathrm{mg})$ plus nedaplatin $(100 \mathrm{mg})$ with a 21-day interval. The patient was treated with $6 \mathrm{MV}$ X-ray IMRT. The total dose of prophylactic radiation in the pelvic lymph drainage area was 50.4 Gy using $1.8 \mathrm{~Gy} / \mathrm{F}$ for $28 \mathrm{~F}$. The radiation doses for the cervical stump and non-central isolated lesion were synchronously increased to $59.92 \mathrm{~Gy}$ using $2.14 \mathrm{~Gy} / \mathrm{F}$ for $28 \mathrm{~F}$ (Fig. 3B).

Following external-beam radiation and chemotherapy, pelvic MRI was performed to examine the effect of treatment on November 24, 2013 (27 months after surgery). No clear swollen shadow was observed at the pelvic lymph nodes, indicating that the nodes were normal. The lesion was ill-defined with its surrounding tissue and had become substantially smaller (Fig. 2C) compared with that on the MRI from August 26, 2013 (Fig. 2B). A gynecological examination revealed vaginal patency, no abnormal secretions, no mucous membrane congestion and a smooth vaginal wall with no palpable nodules. However, a cervical stump biopsy revealed squamous cell hyperplasia, cell heterogeneity and the formation of granulation tissue (Fig. 1E and F). Levels of the tumor marker CA125 were $6.1 \mathrm{U} / \mathrm{ml}$, recovering to a normal level. Following chemotherapy and external-beam radiation, a 

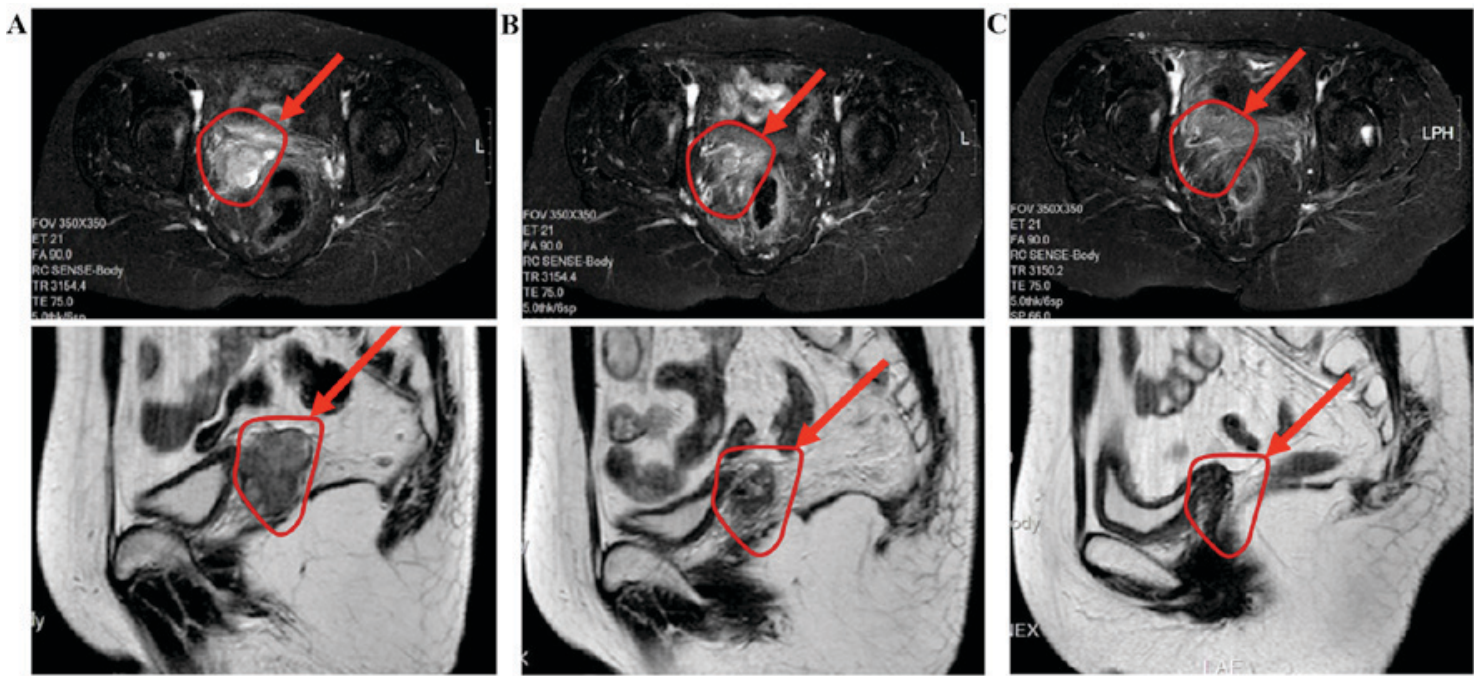

Figure 2. Magnetic resonance imaging prior to and following chemotherapy and external beam radiation therapy. (A) The arrows indicate parametrial isolated tumor lesions prior to chemotherapy. The maximum lesion diameter is $38 \mathrm{~mm}$. (B) Arrows indicate isolated parametrial tumor lesions following 2 cycles of chemotherapy. The maximum diameter is $22 \mathrm{~mm}$ and the tumor size is markedly reduced. (C) Arrows indicate the cross-section of parametrial isolated tumor lesions following external-beam radiation. The size of the isolated tumor size continues to decrease, but a residual lesion remains.
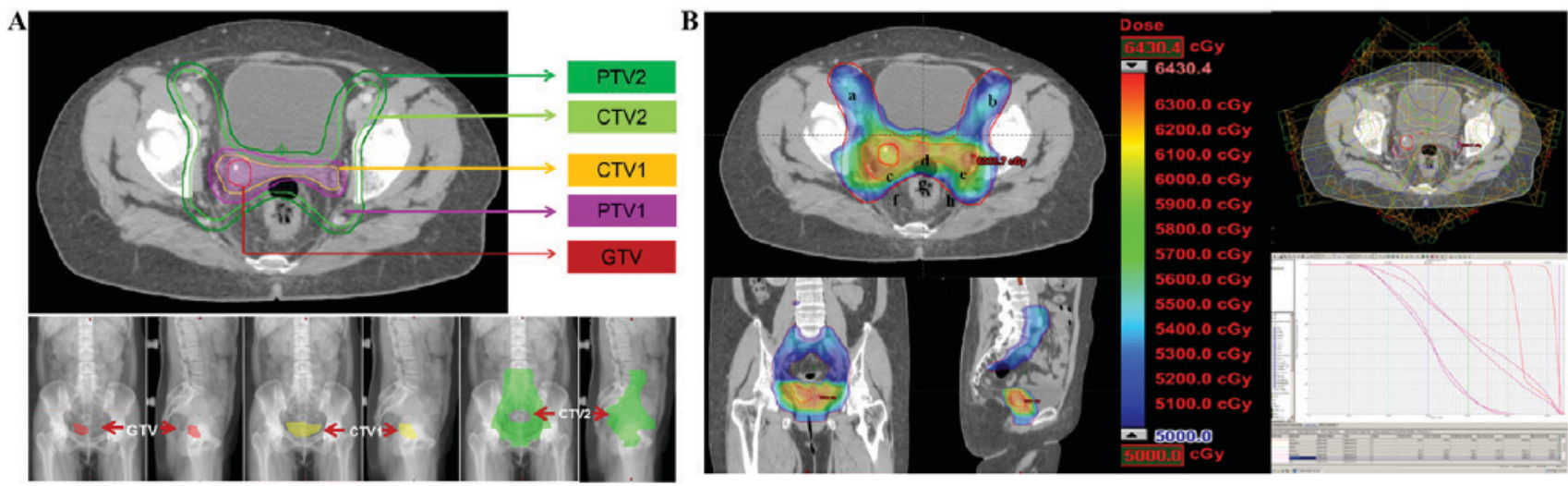

Figure 3. Target area and dose distribution of radiation in recurrent cervical cancer. (A) CTV1 (yellow line) indicates the area of the tumor lesions and cervical stump. PTV1 (purple line) refers to the area $0.8 \mathrm{~mm}$ outside of CTV1. CTV2 (light green line) refers to the lymphatic drainage area. PTV2 (dark green line) refers to the area $0.8 \mathrm{~mm}$ outside of CTV2. GTV (red line) refers to the tumor lesion area. (B) The purple outline (PTV1) indicates the cervical stump and tumor lesions (3, 4 and 5). The total radiation dose is 60 Gy. PTV2 represents the lymphatic drainage area (1 and 2$)$. The total dose is 50.4 Gy. The doses at position 6, 7 and 8 in the anterior wall and perirectal space of the rectum are 52.5, 48.4 and 51.8 Gy, respectively. The dose histogram shows that for PTV1, the exposed $\mathrm{V}_{60}=95 \%$; for PTV2, $\mathrm{V}_{50.4}=95 \%$; the rectum $\mathrm{V}_{50}=38<50 \%$; and the bladder $\mathrm{V}_{50}=27<50 \%$. CTV, clinical target volume; PTV, planned target volume; $\mathrm{V}$, volume exposed to dose in Gy.

residual tumor shadow remained present upon MRI. However, no cancer cells were observed in the cervical stump and the size of the parametrial isolated tumor lesion was markedly reduced. Thus, brachytherapy was continued following the end of external-beam radiotherapy.

On December 2, 2013 (28 months after surgery and 2 weeks after the cessation of external-beam radiation and chemotherapy), the patient was admitted to the Department of Radiotherapy of the Sino-Japanese Friendship Hospital of Jilin University for ${ }^{125}$ I radioactive seed-implantation therapy. A total of $14{ }^{125}$ I seeds were implanted, with seed radiation covering the recurrence area. Seed-implantation treatment plans are shown in Table I. Examinations were performed at 1, 6, 10, 15, 17, 21 and 28 months after implantation (Fig. 4). The images in the first month following implantation showed radiation particle aggregation and effective seed distribution. At 28 months after implantation, a computed tomography (CT) scan revealed that pelvic seed particles were scattered and were not fixed at the designated location. No evident mass had appeared at the location of tumor recurrence (Fig. 4H). The physical condition of the patient was good; the performance status score was 0 points and the patient did not complain of any discomfort.

\section{Discussion}

The present patient was a 47-year-old woman in good health with a performance status score of 1 . The primary disease was diagnosed as International Federation of Gynecology and Obstetrics (FIGO) stage IB1 cervical cancer. The postoperative pathological examination was negative for cancerous tissue, with no vascular invasion and no high-risk factors identified. Therefore, no further treatment was administered following surgery. However, the patient presented with recurrent disease 
23 months after surgery. MRI did not reveal pelvic lymph node metastasis, but a solitary lesion with a maximum diameter of $\sim 38 \mathrm{~mm}$ was found towards the right side of the cervical stump level. A biopsy of the cervical stump revealed squamous cell carcinoma, consistent with the diagnosis of the primary tumor. MRI images did not reveal evident space occupation in the cervical stump (Fig. 2A).

The case was diagnosed as the non-central recurrence of cervical squamous cell carcinoma. The patient had not received chemoradiotherapy following surgery and had only one single recurred lesion without metastasis. According to 2016 NCCN Guidelines for cervical cancer (4), patients with a localized recurrence of cervical cancer following initial treatment may be candidates for radical retreatment. Treatment options include tumor-directed radiotherapy, brachytherapy and/or chemotherapy (if no prior radiotherapy has been administered or the lesion is present outside of the previously treated field) and surgery. As the patient refused surgery, following repeated discussions a treatment regimen of IMRT, chemotherapy and ${ }^{125}$ I seed implantation was chosen.

In clinical practice, the local or regional recurrence of cervical cancer, if it is unresectable and the patient has no history of radiotherapy, can be treated with radiotherapy in combination with platinum-based chemotherapy. Brachytherapy can be used in addition, according to different disease conditions (4).

A phase III clinical trial performed by Monk et al (5) revealed that in FIGO stage IVB recurrent or refractory cervical cancer, a dual-drug regimen of vinorelbine plus cisplatin, gemcitabine plus cisplatin or topotecan plus cisplatin chemotherapy did not produce superior overall survival, PFS or response rates compared with paclitaxel with cisplatin. A phase III clinical trial by Moore et al (6) also supported the dual-drug regimen of paclitaxel plus cisplatin, finding that it produced significantly better PFS than cisplatin monotherapy $(\mathrm{P}<0.001)$. On the basis of these studies and the toxicity of various chemotherapy drugs, docetaxel (as it is similar to paclitaxel, with a reduced likelihood of an allergic reaction) plus nedaplatin (as it is similar to cisplatin, with a reduced likelihood of digestive adverse reactions) was selected for this case. Evaluation following 2 cycles of chemotherapy showed that the size of the tumor had decreased from 38 to $22 \mathrm{~mm}$, confirming the effectiveness of the chemotherapy. Thus, this chemotherapy was applied for a total of 4 cycles.

On the basis of the radiotherapy target for cervical cancer recommended by the US Radiation Therapy Oncology Group and our own clinical practice, the present patient was treated with external-beam radiation using IMRT. The radiation area involved the lymphatic drainage area of the internal iliac, external iliac, presacral and obturator foramen, and a region of the vaginal and parametrial tissue (7). The total dose was 50.4 Gy (Fig. 3B).

As described in Cancer Radiation Therapy by Gu et al (8), the most common radiotherapy treatment for cervical cancer is the combined use of external-beam and intracavitary radiation. The proper coordination of the two techniques can partly compensate for the disadvantages that result from the uneven distribution of a brachytherapy dose. If permitted, the radiation dose can be appropriately increased to complement the insufficient radiation in the parametrial 

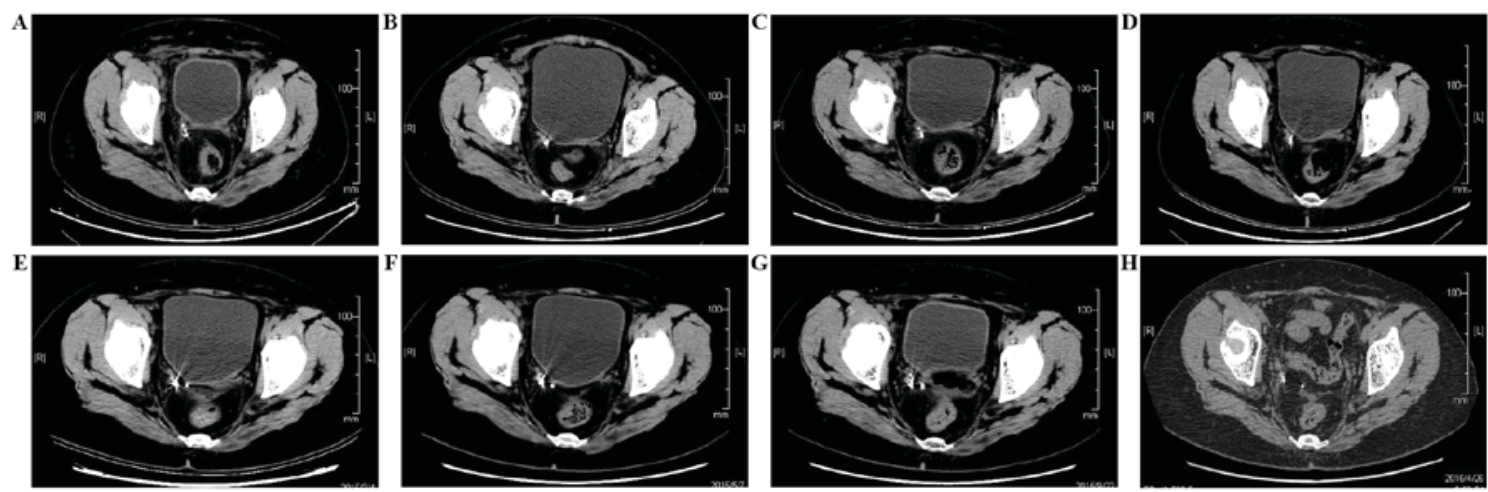

Figure 4. CT scans performed following interstitial ${ }^{125}$ I seed implantation. CT images at (A) 1, (B) 6, (C) 10, (D) 15, (E) 17, (F) 21 and (G) 28 months after ${ }^{125}$ I seed implantation. Arrows indicate the seed distribution in the recurrent tumor. Between months 1 and 17, radioactive particles aggregate and appear to distribute effectively. At 21 months following implantation, the particles are scattered and are not fixed in the designated location, indicating that tumor mass has completely disappeared. CT, computed tomography.

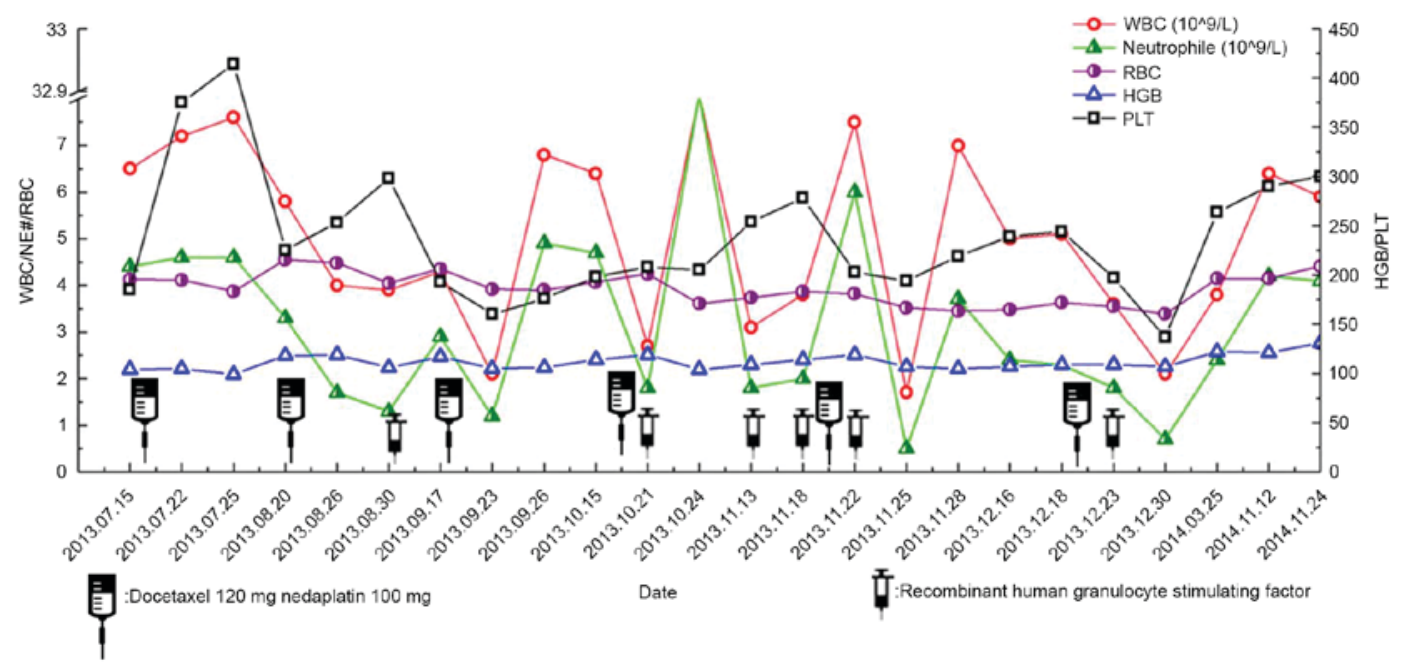

Figure 5. Dynamic changes in WBCs, NEs, RBCs, HGB and PLTs during chemoradiotherapy. The number of WBCs and NEs gradually decreased once chemotherapy began (arrows indicate the start time of a chemotherapy cycle). The number further decreased with further chemotherapy cycles and the increasing extent of bone marrow suppression. WBC, white blood cell; NE, neutrophil; RBC, red blood cell; HGB, hemoglobin; PLT, platelet.

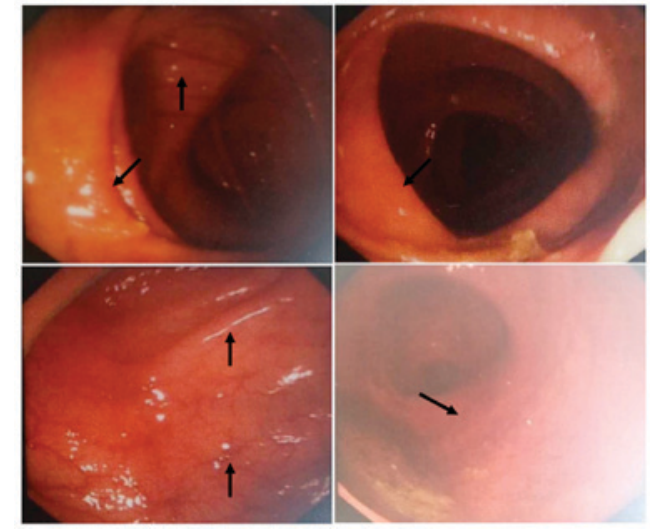

Figure 6. Intestinal mucosal injury 11 months after ${ }^{125}$ I seed implantation. Colonoscopic images reveal local mucosa congestion, edema and poorly defined vasculature in the large intestine.

tissue from intracavitary radiation. Therefore, in uncertain conditions (with a high-risk target, for example), the radiation dose is synchronously increased to $60 \mathrm{~Gy}$ in the tumor area and parametrial tissue using external-beam radiotherapy (Fig. 3B).

In clinical practice, conventional internal radiotherapy primarily includes intracavitary afterloading and interstitial implantation. According to the linear quadratic formula and its derived formula, the basic formula of equivalent transformation of different segmentation scheme is $\mathrm{n}_{2} \mathrm{~d}_{2}\left[1+\mathrm{d}_{2} /(\alpha / \beta)\right]=\mathrm{n}_{1} \mathrm{~d}_{1}\left(1+\mathrm{d}_{1} /(\alpha / \beta)\right]$, with $\mathrm{n}, \mathrm{d}$ and nd refering to the radiation number, dose of one radiation and the total dose, respectively. $\alpha / \beta$ is defined as the tissue-specific $\alpha / \beta$ value. When the prescribed dose is $6 \mathrm{~Gy}$ and the total number of fractions is 5 , the equivalent biological dose is five fractions of $8 \mathrm{~Gy}$, giving a total of $40 \mathrm{~Gy}(\alpha / \beta$ is set as 10$)$. Thus, the total curative dose of external beam radiation plus afterloading should be 85-90 Gy. In other words, once patients have received external-beam radiation, they then require 4-6 treatments of intracavitary afterloading or interstitial implantation. However, this dose could increase the radiation in the bladder posterior wall and the rectal anterior wall, which may increase the likelihood of long-term adverse reactions in these two organs. 
As the present case concerned non-central recurrent cervical cancer with a lesion that deviated from the vaginal center by $\sim 30 \mathrm{~mm}$, conventional intracavitary afterloading would lead to an uneven dose distribution in the target area, perhaps even missing it altogether. If the target area is to be covered by the isodose curve of the dose reference point, the dose and volume of radiation received by the rectum and bladder will be increased. Toita et al (9) reported a three-dimensional interstitial implantation and brachytherapy system, in which the three-dimensional position of an implantation needle was reconstructed, and the gross tumor volume, clinical target volume and radiation dose in the relevant organs were redefined. Consequently, the dose curve of the reference point closely conformed to that of the treatment target, meaning it is possible that the tumor area was accurately radiated by high-dose radiation (9).

According to Sharma et al (10), interstitial implantation-guided afterloading radiotherapy is notably superior to conventional afterloading radiotherapy. In interstitial implantation, the radiation dose to the target area is homogeneously distributed with high coverage, reducing the volume and dose of radiation in normal tissues, including the bladder and rectum. However, when compared with conventional intracavitary afterloading, interstitial implantation-guided afterloading radiotherapy is relatively invasive. The implantation process requires the coordination of multiple implantation needles and repeated $\mathrm{CT}$ scans to confirm and adjust the location and depth of the needles. The total treatment plan requires 4-6 implantation procedures, which increases the risk of bleeding, infection and radiation hazards, and the possibility of iatrogenic tumor seeding and metastasis.

${ }^{125}$ I seed implantation is a minimally invasive, widely used treatment that has unique advantages in the treatment of locally advanced tumors $(11,12)$. In the US, radioactive seed implantation has been used as the standard treatment for early prostate cancer (13). However, the use of ${ }^{125}$ I seed implantation has rarely been reported for the treatment of non-central recurrent cervical cancer. The ${ }^{125}$ I seed has a half-life of 59.6 days and has similar biological characteristics to hyperfractionated radiotherapy; ${ }^{125}$ I seeds continuously irradiate cells in different phases of the cell cycle through the continuous release of low-energy $\gamma$-rays, increasing their sensitivity to radiation and promoting recurrent cervical cancer cell death (14).

Once the size of the tumor is confirmed by a CT scan and the treatment plan is decided upon using a three-dimensional treatment-planning system, the radioactive seeds are implanted into the tumor using ultrasound-guidance. The photons released from the radioactive seeds produce continuous, low-dose radiation at the position of the lesion (14). This treatment method achieves the purpose of conventional radiotherapy, while protecting the surrounding normal organs as much as possible.

Although the initial radiation dose-rate of the ${ }^{125}$ I seeds was low in the present study, it did not cause uncontrolled tumor growth, as the present case was of squamous cell carcinoma, in which tumor cell proliferation is slow. In the present study, the patient and her family were provided detailed information on the treatment regimen and consented to interstitial ${ }^{125} \mathrm{I}$ seed-implantation therapy following the end of external-beam radiotherapy and chemotherapy.
The efficacy of the treatment was evaluated using MRI or CT images, based on the Response Evaluation Criteria In Solid Tumors (15). Complete remission (CR) refers to the disappearance of all target lesions, the presence of no new lesions, and levels of tumor markers within the normal range. All these should be maintained for at least 4 weeks. Partial remission (PR) is achieved when the sum of the maximum diameters of target lesions are reduced by $\geq 30 \%$ for at least 4 weeks. A pelvic CT scan was examined 1 month after seed implantation, at which point partial remission had been achieved. A further CT scan 21 months after the treatment revealed there was CR. Evaluation of treatment efficacy was performed every 3 months using pelvic MRI or CT scans and no progression was observed (Fig. 4). The latest evaluation of the patient revealed that the PFS time had reached 33 months.

Different degrees of bone marrow suppression occurred during treatment, including four incidences of grade 1 bone marrow suppression, two incidences of grade 2 and two incidences of grade 3. No incidences of grade 4 occurred (Fig. 5). Following the administration of recombinant human granulocyte colony stimulating factor, bone marrow suppression was improved. No infection event occurred.

At 11 months after seed implantation, the patient suffered diarrhea and a grade 2 gastrointestinal adverse effect. A colonoscopy revealed mucosa congestion, edema and poorly defined vasculature in the large intestine (Fig. 6). After 1 week of administration of intestinal mucosal protective agents and active symptomatic treatment, these symptoms completely disappeared.

Data from the present study show that IMRT combined with ${ }^{125}$ I seed implantation, as a supplement for the treatment of recurrent cervical cancer, can achieve the desired curative effect. As the patient in the present study had similar characteristics to primary cervical cancer, it may have the potential for use as a reliable radical radiotherapy in newly diagnosed cervical cancer.

\section{References}

1. Torre LA, Bray F, Siegel RL, Ferlay J, Lortet-Tieulent J and Jemal A. Global cancer statistics, 2012. CA Cancer J Clin 65: 87-108, 2015.

2. Goncalves A, Fabbro M, Lhommé C, Gladieff L, Extra JM, Floquet A, Chaigneau L, Carrasco AT and Viens P: A phase II trial to evaluate gefitinib as second- or third-line treatment in patients with recurring locoregionally advanced or metastatic cervical cancer. Gynecol Oncol 108: 42-46, 2008.

3. Elit LM and Hirte H: Management of advanced or recurrent cervical cancer: Chemotherapy and beyond. Expert Rev Anticancer Ther 14: 319-332, 2014.

4. National Comprehensive Cancer Network (NCCN): NCCN Clinical Practice Quidelines in Oncology: Cervical Cancer. version 2. 2015. http://www.ncen.org/professionals/physician gls/f_guidelines.asp. Accessed March 11, 2017.

5. Monk BJ, Sill MW, McMeekin DS, Cohn DE, Ramondetta LM, Boardman $\mathrm{CH}$, Benda J and Cella D: Phase III trial of four cisplatin-containing doublet combinations in stage IVB, recurrent, or persistent cervical carcinoma: A gynecologic oncology group study. J Clin Oncol 27: 4649-4655, 2009.

6. Moore DH, Blessing JA, McQuellon RP, Thaler HT, Cella D, Benda J, Miller DS, Olt G, King S, Boggess JF and Rocereto TF: Phase III study of cisplatin with or without paclitaxel in stage IVB, recurrent, or persistent squamous cell carcinoma of the cervix: A gynecologic oncology group study. J Clin Oncol 22: 3113-3119, 2004. 
7. Japan Clinical Oncology Group; Toita T, Ohno T, Kaneyasu Y, Uno T, Yoshimura R, Kodaira T, Furutani K, Kasuya G, Ishikura S, et al: A consensus-based guideline defining the clinical target volume for pelvic lymph nodes in external beam radiotherapy for uterine cervical cancer. Jpn J Clin Oncol 40: 456-463, 2010

8. Gu X, Yin W, Hu Y, et al: Tumor radiation therapy. Pecking Union Medical College Press, Beijing, 2007.

9. Toita T, Kitagawa R, Hamano T, Umayahara K, Hirashima Y, Aoki Y, Oguchi M, Mikami M and Takizawa K; Cervical Cancer (Vulva Cancer) Committee of Japanese Gynecologic Oncology Group (JGOG): Phase II study of concurrent chemoradiotherapy with high-dose-rate intracavitary brachytherapy in patients with locally advanced uterine cervical cancer: Efficacy and toxicity of a low cumulative radiation dose schedule. Gynaecol Oncol 126: 211-216, 2012.

10. Sharma DN, Subramani V, Rath GK, Ganesh T, Julka PK, Jyothi Basu KS, Bahl A and Gopishankar N: Interstitial brachytherapy guided intensity modulated radiation therapy in cervical cancer: A dosimetric study. Int J Radiation Oncol Biol Phys 69 (Suppl) S731-S732, 2007.
11. Shi L, Wu C, Wu J, Zhou W, Ji M, Zhang H, Zhao J, Huang Y, Pei H, Li Z, et al: Computed tomography-guided permanent brachytherapy for locoregional recurrent gastric cancer. Radiat Oncol 7: 114, 2012.

12. Wang J, Jiang Y, Li J, Tian S, Ran W and Xiu D: Intraoperative ultrasound-guided iodine-125 seed implantation for unresectable pancreatic carcinoma. J Exp Clin Cancer Res 28: 88, 2009.

13. Nag S, Ellis RJ, Merrick GS, Bahnson R, Wallner K and Stock R; American Brachytherapy Society: American brachytherapy society recommendations for reporting morbidity after prostate brachytherapy. Int J Radiat Biol Phys 54: 462-470, 2002.

14. Yao L, Wang J, Jiang Y, Li J, Lin L, Ran W and Liu C: Permanent interstitial 125I seed implantation as a salvage therapy for pediatric recurrent or metastatic soft tissue sarcoma after multidisciplinary treatment. World J Surg Oncol 13: 335, 2015.

15. Eisenhauer EA, Therasse P, Bogaerts J, Schwartz LH, Sargent D, Ford R, Dancey J, Arbuck S, Gwyther S, Mooney M, et al: New response evaluation criteria in solid tumours: Revised RECIST guideline (version 1.1). Eur J Cancer 45: 228-247, 2009. 